\title{
Onset of volatile recycling into the mantle determined by xenon anomalies
}

\section{S. Péron ${ }^{1 *}$, M. Moreira ${ }^{1}$}

Abstract

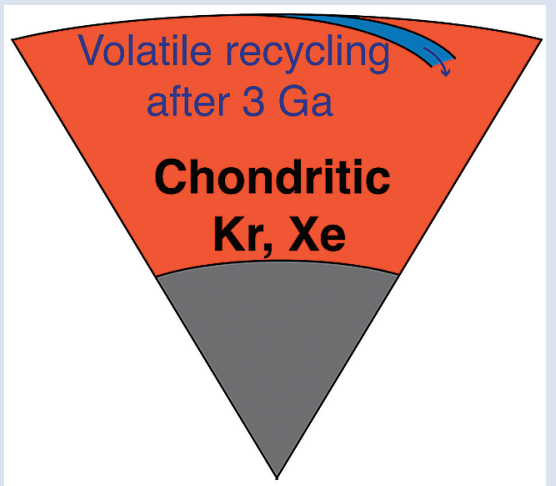

Noble gases serve as unique tracers of the origin and evolution of Earth's volatile reservoirs owing to their inert nature and contribution from extinct and extant radioactivities. However, noble gases are low in abundance relative to many other elements, particularly in the Earth's mantle. Additionally, mantle-derived samples show large post-eruptive atmospheric contamination, rendering the determination of the primary mantle composition challenging. The sources of mantle krypton and xenon remain debated due to their partially resolvable excess, if any, relative to the atmosphere. Atmospheric noble gases also appear to be recycled into the mantle via subduction, progressively overprinting the initial mantle signature. Here we develop a new protocol to accumulate non-contaminated mantle-derived xenon, in particular the low abundant ${ }^{124-126-128} \mathrm{Xe}$. The results show the highest excesses in ${ }^{124-126-128}$ Xe ever measured in the mantle relative to the atmosphere and point toward a chondritic origin for mantle xenon. The fissiogenic isotopes ${ }^{131-132-134-136}$ Xe allow the onset of efficient xenon recycling in the mantle to be constrained at around 3 Gyr ago, implying that volatile recycling before 3 Ga would have been negligible.

Received 31 July 2018 | Accepted 24 November 2018 | Published 20 December 2018

\section{Letter}

The inert noble gases ( $\mathrm{He}, \mathrm{Ne}, \mathrm{Ar}, \mathrm{Kr}, \mathrm{Xe})$ serve as an invaluable tool for constraining the volatile origin and evolution of terrestrial planetary reservoirs. Among them, Xe with its nine isotopes is an ideal tracer of both volatile origin and mantle evolution because all of its isotopes represent distinct geochemical signatures (Kunz et al., 1998; Moreira et al., 1998; Caffee et al., 1999; Holland and Ballentine, 2006; Pujol et al., 2011; Mukhopadhyay, 2012; Tucker et al., 2012; Parai and Mukhopadhyay, 2015, 2018; Avice et al., 2017; Marty et al., 2017). Indeed, ${ }^{124-126-128-130} \mathrm{Xe}$ are non-radiogenic, stable isotopes whereas ${ }^{129} \mathrm{Xe}$ is radiogenic (decay product of the now extinct ${ }^{129}$ I radioactivity) and ${ }^{131-132-134-136} \mathrm{Xe}$ are fissiogenic, both deriving from the fission of the now extinct ${ }^{244} \mathrm{Pu}$ nuclide (half life $80 \mathrm{Myr}$ ) and the still alive ${ }^{238} \mathrm{U}$ nuclide.

Many studies have focused on the radiogenic and fissiogenic Xe compositions of the Earth's mantle from analyses of mid-ocean ridge basalts (MORBs) (Kunz et al., 1998; Moreira et al., 1998; Parai et al., 2012; Tucker et al., 2012; Parai and Mukhopadhyay, 2015), plume-influenced back-arc basin basalts (Petö et al., 2013), oceanic island basalts (OIBs) (Poreda and Farley, 1992; Trieloff et al., 2000, 2002; Mukhopadhyay, 2012), $\mathrm{CO}_{2}$ well gases (Caffee et al., 1999; Holland and Ballentine, 2006; Holland et al., 2009) and thermal springs (Caracausi et al., 2016; Moreira et al., 2018) because these isotopes are relatively abundant. However, ${ }^{124-126-128}$ Xe isotopes are very rare and thus extremely difficult to measure. Initial studies measured the abundances of ${ }^{124-126-128} \mathrm{Xe}$ in mantle-derived samples but proved these to be unresolvable from that of the atmosphere (Kunz et al., 1998). The only successful attempts to resolve ${ }^{124-126-128} \mathrm{Xe}$ excess have been during the analyses of $\mathrm{CO}_{2}$ well gases and thermal springs (Caffee et al., 1999; Holland and Ballentine, 2006; Caracausi et al., 2016). However, recycling of atmospheric xenon via subduction into the convecting mantle effectively overprints and erases the primitive mantle signature, as more than $80 \%$ of Xe in the mantle is assumed to be derived through the subduction of oceanic lithosphere (Caffee et al., 1999; Holland and Ballentine, 2006; Mukhopadhyay, 2012; Parai and Mukhopadhyay, 2015). Consequently it has long been assumed that anomalies of ${ }^{124-126-128}$ Xe were undetectable in basaltic glasses (Trieloff et al., 2000).

A second issue surrounding the determination of ${ }^{124-126-}$ ${ }^{128} \mathrm{Xe}$ excess is that basaltic glasses are highly susceptible to post-eruptive contamination by air (Ballentine and Barfod, 2000). Typically air can enter glass samples and be hosted within small cracks and open vesicles (Ballentine and Barfod, 2000), so that when samples are crushed, this air component mixes with mantle gases from intact bubbles, rendering determination of Xe anomalies even more difficult if not impossible.

Here a new protocol is developed to accumulate air-free xenon from intact glass vesicles of MORBs and determine whether ${ }^{124-126-128} \mathrm{Xe}$ anomalies can be detected in the convecting mantle. To this end, the gas-rich popping rock $2 \pi \mathrm{D} 43$ sample is analysed, which has been extensively studied

1. Institut de Physique du Globe de Paris - Sorbonne Paris Cité, UMR CNRS 7154, Université Paris Diderot. 1 Rue Jussieu, 75005, Paris, France

* Corresponding author (email: peron@ipgp.fr) 
(Burnard et al., 1997; Kunz et al., 1998; Moreira et al., 1998). The new protocol consists of sequential crushing steps, with the neon composition of each step being used as a monitor of post-eruptive atmospheric contamination, as Ne displays distinct atmospheric and mantle compositions $\left({ }^{20} \mathrm{Ne} /{ }^{22} \mathrm{Ne}\right.$ ratios of 9.8 and 12.5 respectively). If the ${ }^{20} \mathrm{Ne} /{ }^{22} \mathrm{Ne}$ ratio is higher than 11.8, then the heavy noble gases from each crush step are accumulated on activated charcoal (Fig. S-1 and Supplementary Information).

The results are shown in Figure 1 and in Tables S-1 and $\mathrm{S}-2$. The ${ }^{129} \mathrm{Xe} /{ }^{130} \mathrm{Xe}$ ratio of the accumulated gas is $7.41 \pm 0.03$

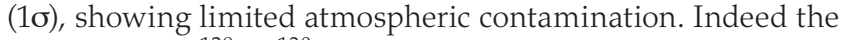
upper mantle ${ }^{129} \mathrm{Xe} /{ }^{130} \mathrm{Xe}$ ratio is assumed to be 7.6 based on previous popping rock $2 \pi \mathrm{D} 43$ data (Moreira et al., 1998). The measured xenon isotopic ratios are hence corrected for this limited atmospheric contamination (17\%), assuming that the uncontaminated ${ }^{129} \mathrm{Xe} /{ }^{130} \mathrm{Xe}$ ratio is 7.6 (Table S-1) and the two data (measured and corrected) are indicated in Figure 1. The high measured ${ }^{129} \mathrm{Xe} /{ }^{130} \mathrm{Xe}$ ratio (7.41) allows checking that the new protocol is very efficient for determining the mantle xenon composition with almost no atmospheric contamination (Fig. S-2).
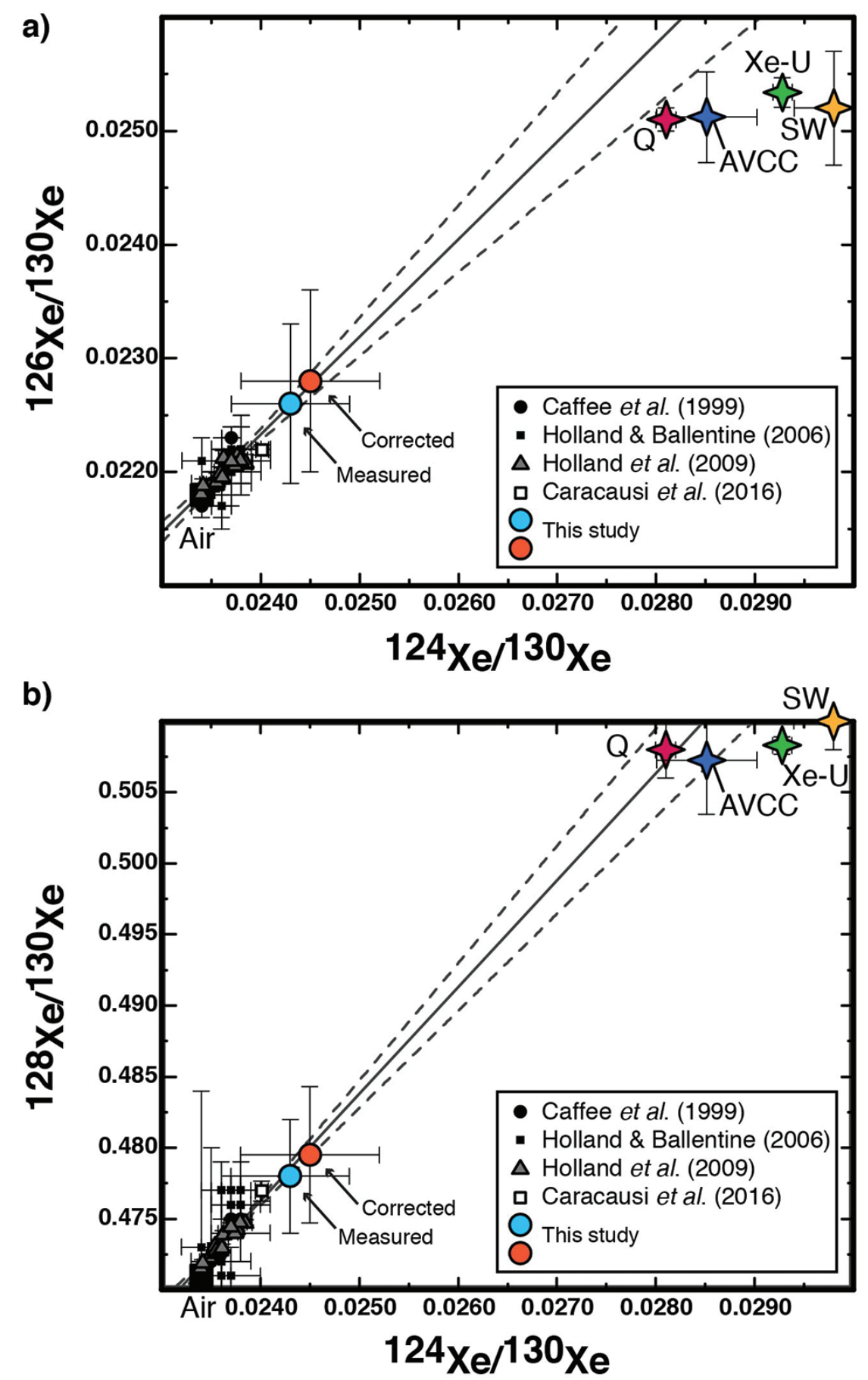

Figure 1 Light xenon isotopic compositions for popping rock $2 \pi D 43$. Measured data (blue dot) and corrected data for atmospheric contamination (orange dot) with a ${ }^{129} \mathrm{Xe} /{ }^{130} \mathrm{Xe}$ ratio of 7.6: (a) ${ }^{126} \mathrm{Xe} /{ }^{130} \mathrm{Xe}$ and (b) ${ }^{128} \mathrm{Xe} /{ }^{130} \mathrm{Xe}$ versus ${ }^{124} \mathrm{Xe} /{ }^{130} \mathrm{Xe}$. For comparison, data of $\mathrm{CO}_{2}$ well gases from Caffee et al. (1999) (black points), Holland and Ballentine (2006) (black square) and Holland et al. (2009) (grey triangles), and thermal springs from Caracausi et al. (2016) (green square) and Moreira et al. (2018) (red square) are shown. The solid line is a fit of the data, except that of Holland and Ballentine (2006). The dotted lines indicate the $95 \%$ confidence interval. Phase $\mathrm{Q}$ (Busemann et al., 2000), Solar Wind SW (Meshik et al., 2014), Xe-U and AVCC (Pepin, 2003). These new data suggest a chondritic origin (Phase Q or AVCC) for upper mantle Xe. 
An excess in ${ }^{124-126-128} \mathrm{Xe}$ is clearly observed for sample popping rock $2 \pi \mathrm{D} 43$. This excess is greater than the previously measured excess observed in $\mathrm{CO}_{2}$ well gases (Fig. 1), and is the first significant ${ }^{124-126-128} \mathrm{Xe}$ excess measured in a MORB sample. A linear fit through the data suggests a chondritic xenon composition (Phase Q, the main carrier of heavy noble gases in chondrites (Busemann et al., 2000) or AVCC for Average Carbonaceous Chondrites (Pepin, 2003)) for the initial mantle (Fig. 1) rather than Solar Wind. This seems also to be the case for $\mathrm{Kr}$ (Fig. S-3 and Table S-2). A chondritic origin of mantle Xe (Caracausi et al., 2016) and Kr (Holland et al., 2009) was first suggested based on analyses of $\mathrm{CO}_{2}$ well gases and thermal springs. However, the excesses in ${ }^{124-126-128} \mathrm{Xe}$ measured for the Eifel spring (Caracausi et al., 2016) remain questionable given that strong atmospheric contamination is needed to explain the Eifel heavy Xe pattern (Moreira et al., 2018) ( ${ }^{131-136}$ Xe; Fig. 2). Therefore, these new data suggest a chondritic origin for upper mantle heavy noble gases. It is however not possible to distinguish between Phase $Q$ and AVCC, contrary to the suggestion that mantle $\mathrm{Kr}$ and Xe were derived from material similar to AVCC (Holland et al., 2009). Comets would not have contributed significantly to mantle xenon contrary to atmospheric xenon (Marty et al., 2017, and discussion below).
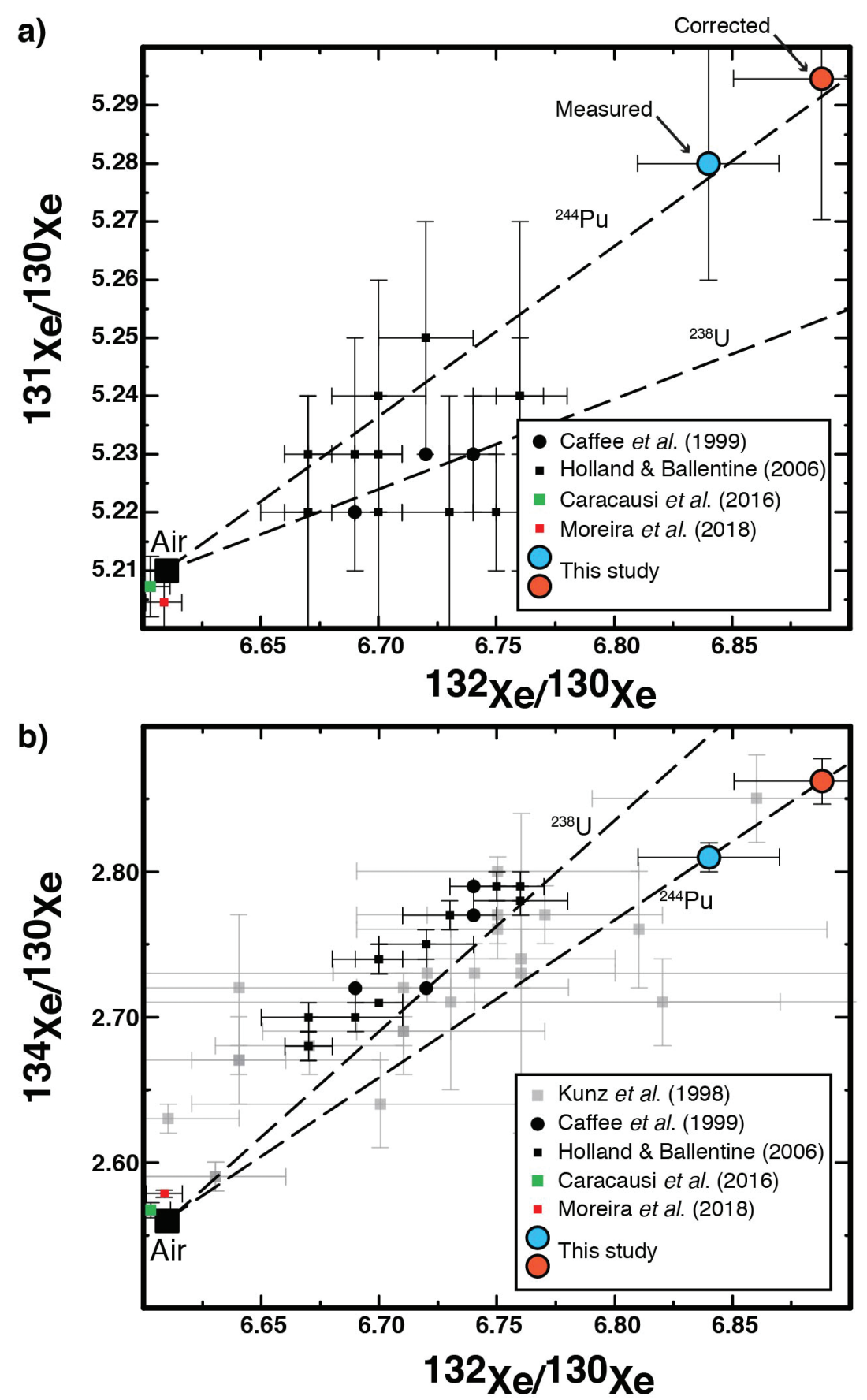

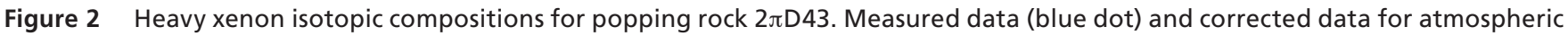
contamination (orange dot) with a ${ }^{129} \mathrm{Xe} /{ }^{130} \mathrm{Xe}$ ratio of 7.6 : (a) ${ }^{131} \mathrm{Xe} /{ }^{130} \mathrm{Xe}$ and $(\mathbf{b}){ }^{134} \mathrm{Xe} /{ }^{130} \mathrm{Xe}$ versus ${ }^{132} \mathrm{Xe} /{ }^{130} \mathrm{Xe}$. Data for sample $2 \pi \mathrm{D} 43$ from Kunz et al. (1998), for $\mathrm{CO}_{2}$ well gases from Caffee et al. (1999) (black points), Holland and Ballentine (2006) (black square), and thermal springs from Caracausi et al. (2016) (green square) and Moreira et al. (2018) (red square) are shown. The trends of pure ${ }^{244} \mathrm{Pu}-$ and ${ }^{238} \mathrm{U}$-derived xenon productions are also indicated. The source of sample $2 \pi \mathrm{D} 43$ seems to be more influenced by Pu-derived Xe than U-derived Xe, contrary to other MORBs (Tucker et al., 2012; Parai and Mukhopadhyay, 2015). 
Recycling of atmospheric Xe is required to explain the present day mantle ${ }^{124-126-128}$ Xe composition (Fig. 1) (Caffee et al., 1999; Holland and Ballentine, 2006; Mukhopadhyay, 2012; Parai and Mukhopadhyay, 2015; Caracausi et al., 2016). The fissiogenic mantle ${ }^{131-136} \mathrm{Xe}$ (Fig. 2) is considered to reflect mixing of four components, namely initial Xe, recycled atmospheric $\mathrm{Xe}, \mathrm{Pu}$-derived $\mathrm{Xe}$ and uranium-derived $\mathrm{Xe}$. The contributions of each of these four components was deconvoluted in mantle-derived samples (Caffee et al., 1999; Mukhopadhyay, 2012; Tucker et al., 2012; Petö et al., 2013; Parai and Mukhopadhyay, 2015; Caracausi et al., 2016), in an attempt to determine the ratio of $\mathrm{Pu}$ - to U-derived $\mathrm{Xe}$ in mantle sources and so better constrain their degassed states, a higher $\mathrm{Pu}-$ to $\mathrm{U}$-derived Xe ratio reflecting a less degassed source due to the fact that ${ }^{244} \mathrm{Pu}$ is now extinct. The new data shown in Figures 2 and S-4 suggest that the source of the popping rock $2 \pi \mathrm{D} 43$ is more heavily influenced by Pu-derived Xe than U-derived Xe, contrary to other MORBs (Tucker et al., 2012; Parai and Mukhopadhyay, 2015).

Incorporation of atmospheric Xe into the mantle is further complicated by the fact that the Xe composition of the atmosphere has changed over time (Pujol et al., 2011; Avice et al., 2017, 2018; Bekaert et al., 2018), starting with the composition of the primordial U-Xe component (Pepin, 2003) and reaching the present day composition, enriched in heavy isotopes by about 40 per mille $\mathrm{u}^{-1}$ compared with $\mathrm{U}-\mathrm{Xe}$, around 2 Gyr ago (Avice et al., 2018). The U-Xe component was first theoretically suggested to explain the Xe isotopic pattern of the atmosphere (Pepin, 2003) and measurements of Xe in comet $67 \mathrm{P} / \mathrm{C}-\mathrm{G}$ reveal that U-Xe could be a mixture of chondritic and cometary volatiles (Marty et al., 2017). Xenon loss from the atmosphere to the outer space accompanied by isotopic fractionation could explain this evolution even if the physical process leading to this loss is still debated (Avice et al., 2018). Such an evolution renders a precise deconvolution of the contributions of the aforementioned four components to the mantle Xe array very difficult, because the isotopic composition of recycled atmospheric xenon strongly varied over time, whilst the mantle has continuously lost $\mathrm{Pu}$ - and U-derived Xe alongside initial Xe through degassing. Therefore, results from previous studies (Caffee et al., 1999; Mukhopadhyay, 2012; Tucker et al., 2012; Petö et al., 2013; Parai and Mukhopadhyay, 2015; Caracausi et al., 2016) may be biased and may not precisely estimate the ratio of $\mathrm{Pu}$ - to $\mathrm{U}$-derived $\mathrm{Xe}$ in the mantle. A recent study modelled the xenon isotopic composition of the mantle taking into account degassing and regassing with an evolving xenon atmospheric composition and found that xenon recycling would have been significant after 2.5 Gyr ago (Parai and Mukhopadhyay, 2018).

Here, the limits on the mantle composition without regassing and the limit on the weighted average age of recycled air are determined, considering the evolution of the Xe atmospheric composition. First, the evolution of the Xe atmospheric isotopic ratios are derived based on a power law for the Xe fractionation factor (Bekaert et al., 2018). The data corrected for shallow atmospheric contamination (Fig. 1 and Table S-1) is considered in a ${ }^{128} \mathrm{Xe} /{ }^{130} \mathrm{Xe} v s$. $\mathrm{R}$ space, where $\mathrm{R}$ represents the ratio of one fissiogenic Xe isotope $\left({ }^{131,132,134,136} \mathrm{Xe} /{ }^{130} \mathrm{Xe}\right.$; Fig. $3)$. The corrected fissiogenic data are thus extrapolated to an initial chondritic (Phase Q) ${ }^{128} \mathrm{Xe} /{ }^{130} \mathrm{Xe}$ for the mantle, as the data in this contribution point to a chondritic origin, considering mixing with the atmosphere to determine the mantle $\mathrm{R}$ ratio before recycling. This mixing relationship is calculated for different air compositions through time (Fig. 3), with the minimum $\mathrm{R}$ ratio being obtained if only present day air has been recycled and the maximum ratio represents the scenario if only ancient air has been recycled (Figs. S-5, S-6 and Supplementary Information).

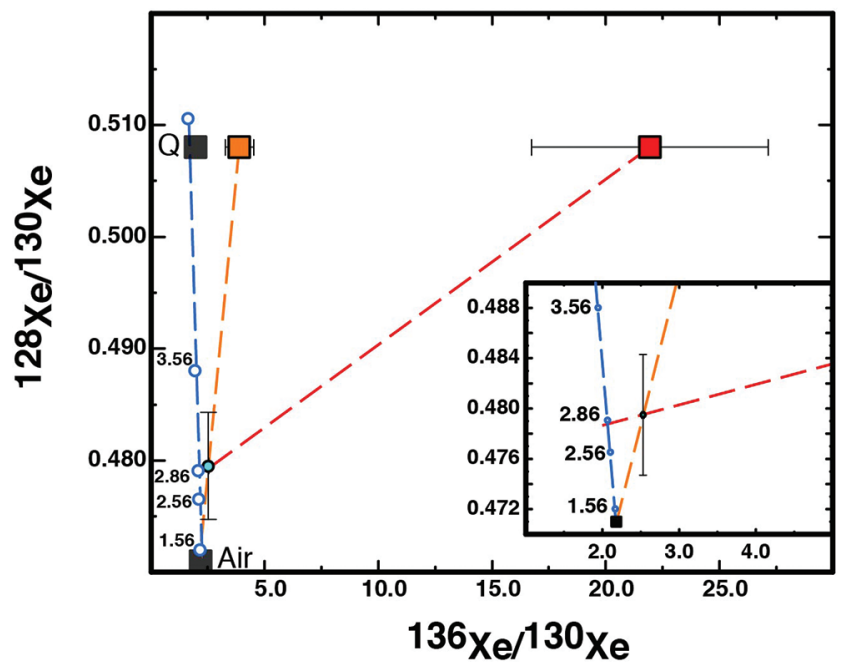

Figure 3 Determination of the maximum average age of recycled atmosphere in the mantle. The evolution of the xenon atmospheric composition is represented with the blue dashed line with the numbers indicating the time in Gyr (a power law was considered; Bekaert et al., 2018). The corrected data for shallow atmospheric contamination is shown (light blue dot). The minimum (3.9 $\pm 0.6(1 \sigma)$; orange square) and maximum (21.9 \pm 5.2 $(1 \sigma)$; red square) ${ }^{136} \mathrm{Xe} /{ }^{130} \mathrm{Xe}$ ratios in the mantle before recycling of atmospheric xenon are calculated considering mixing with air (orange and red dashed lines) and that the initial ${ }^{128} \mathrm{Xe} /{ }^{130} \mathrm{Xe}$ is chondritic (Phase Q; Busemann et al., 2000). The red line suggests that recycling of atmospheric xenon could have been effective only since $2.8 \pm 0.3(1 \sigma) \mathrm{Gyr}$ ago. Otherwise, unreasonable values of ${ }^{136} \mathrm{Xe} /{ }^{130} \mathrm{Xe}$ ratios are obtained.

The maximum air-corrected mantle ${ }^{131-136} \mathrm{Xe} /{ }^{130} \mathrm{Xe}$ ratios (Figs. S-5, S-6) are determined for a recycling of air at $2.8 \pm 0.3(1 \sigma)$ Gyr ago (Fig. 3). This represents a limit on the weighted average age of recycled atmosphere retained in the mantle. Ancient atmospheric gas could have been recycled but not enough to draw this average above $2.8 \mathrm{Ga}$, otherwise the slopes of the mixing lines between ancient air and the corrected data would be negative (Figs. 3, S-7).

Modelling results suggest that effective recycling of Xe, and likely of other noble gases, could not have started before $2.8 \mathrm{Ga}$. This result is consistent with the mantle evolving to a net regassing regime after 2.5 Ga (Parai and Mukhopadhyay, 2018). This time limit places important constraints on volatile evolution. Either it is consistent with studies that showed that subduction on Earth started around 3 Gyr ago (e.g., Dhuime et al., 2012), or subduction started earlier (e.g., Harrison et al., 2005) but volatile recycling was only efficient from $3 \mathrm{Ga}$. In the latter case, the delayed efficient recycling of volatiles could be related to the secular cooling of the Earth as it was suggested that noble gases and other volatiles such as water would mainly be recycled into the mantle through cold subduction zones (van Keken et al., 2011; Parai and Mukhopadhyay, 2015; Smye et al., 2017), while it is likely that early subduction was dominated by hot slabs (van Keken et al., 2011).

\section{Acknowledgements}

We wish to thank Rita Parai and an anonymous reviewer for their constructive comments that helped improving the paper as well as Cin-Ty Lee for editorial handling. We are also very grateful to Guillaume Avice and Edward Inglis for fruitful discussions on the first version of this manuscript. The authors acknowledge the Labex UnivEarthS for its financial support. 
S.P. also wants to acknowledge the financial support from the L'Oréal-UNESCO For Women in Science France 2017 fellowship. This is IPGP contribution number 3994.

Editor: Cin-Ty Lee

\section{Additional Information}

Supplementary Information accompanies this letter at http:// www.geochemicalperspectivesletters.org/article1833.

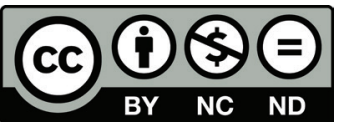

This work is distributed under the Creative Commons Attribution Non-Commercial No-Derivatives 4.0 License, which permits unrestricted distribution provided the original author and source are credited. The material may not be adapted (remixed, transformed or built upon) or used for commercial purposes without written permission from the author. Additional information is available at http://www.geochemicalperspectivesletters.org/ copyright-and-permissions

Cite this letter as: Péron, S., Moreira, M. (2018) Onset of volatile recycling into the mantle determined by xenon anomalies. Geochem. Persp. Let. 9, 21-25.

\section{References}

Avice, G., Marty, B., Burgess, R. (2017) The origin and degassing history of the Earth's atmosphere revealed by Archean xenon. Nature Communications 8, 15455 .

Avice, G., Marty, B., Burgess, R., Hofmann, A., Philippot, P. ZAHNLE, K., ZAKHAROV, D. (2018) Evolution of atmospheric xenon and other noble gases inferred from Archean to Paleoproterozoic rocks. Geochimica et Cosmochimica Acta 232, 82-100.

BALlentine, C., BARFOD, D. (2000) The origin of air-like noble gases in MORB and OIB. Earth and Planetary Science Letters 180, 39-48.

Bekaert, D.V., Broadley, M.W., Delarue, F., Avice, G., Robert, F., MARTY, B. (2018) Archean kerogen as a new tracer of atmospheric evolution: Implications for dating the widespread nature of early life. Science Advances 4, doi: 10.1126/sciadv.aar2091.

Burnard, P., Graham, D., TURner, G. (1997) Vesicle-specific noble gas analyses of "popping rock": implications for primordial noble gases in the Earth. Science 276, 568-571.

Busemann, H., Baur, H., Wieler, R. (2000) Primordial noble gases in "phase $\mathrm{Q}$ " in carbonaceous and ordinary chondrites studied by closedsystem stepped etching. Meteoritics and Planetary Science 35, 949-973.

Caffee, M.W., Hudson, G.P., Velsko, C., Huss, G.R., Alexander, E.C., CHIVAs, R. (1999) Primordial Noble Gases from Earth's Mantle: Identification of Primitive Volatile Component. Science 285, 2115-2118.

Caracausi, A., Avice, G., Burnard, P.G., Füri, E., Marty, B. (2016) Chondritic xenon in the Earth's mantle. Nature 533, 82-85.

Dhuime, B., Hawkesworth, C.J., CAwood, P.A., Storey, C.D. (2012) A Change in the Geodynamics of Continental Growth 3 Billion Years Ago. Science 335, 1334

Harrison, T.M., Blichert-Toft, J., Müller, W., Albarede, F., Holden, P., MoJzsis, S.J. (2005) Heterogeneous Hadean Hafnium: Evidence of Continental Crust at 4.4 to $4.5 \mathrm{Ga}$. Science 310, 1947

Holland, G., Ballentine, C.J. (2006) Seawater subduction controls the heavy noble gas composition of the mantle. Nature 441, 186-191.

Holland, G., Cassidy, M., Ballentine, C.J. (2009) Meteorite Kr in Earth's Mantle Suggests a Late Accretionary Source for the Atmosphere. Science 326, 1522-1525.

Kunz, J., Staudacher, T., Allègre, C.J. (1998) Plutonium-Fission Xenon Found in Earth's Mantle. Science 280, 877-880.

Marty, B., AltwegG, K., Balsiger, H., Bar-Nun, A., Bekaert, D.V., Berthelier, J.J., Bieler, A., Briois, C., Calmonte, U., Combi, M., De Keyser, J., Fiethe, B., Fuselier, S.A., Gasc, S., Gombosi, T.I., Hansen, K.C., HÄSsIG, M., JÄCKEL, A., Kopp, E., Korth, A., Le Roy, L., Mall, U., Mousis, O., Owen, T., Rème, H., Rubin,
M., SÉmon, T., Tzou, C.Y., Waite, J.H., Wurz, P. (2017) Xenon isotopes in 67P/Churyumov-Gerasimenko show that comets contributed to Earth's atmosphere. Science 356, 1069-1072.

Meshik, A., Hohenberg, C., Pravdivtseva, O., Burnett, D. (2014) Heavy noble gases in solar wind delivered by Genesis mission. Geochimica et Cosmochimica Acta 127, 326-347.

MoreirA, M., Kunz, J., Allègre, C.J. (1998) Rare gas systematics on popping rock : estimates of isotopic and elemental compositions in the upper mantle. Science 279, 1178-1181.

Moreira, M., Rouchon, V., Muller, E., Noirez, S. (2018) The xenon isotopic signature of the mantle beneath Massif Central. Geochemical Perspectives Letters 6, 28-32.

MUKHOPADHYAY, S. (2012) Early differentiation and volatile accretion recorded in deep mantle Neon and Xenon. Nature 486, 101-104.

Parai, R., Mukhopadhyay, S. (2015) The evolution of MORB and plume mantle volatile budgets: Constraints from fission Xe isotopes in Southwest Indian Ridge basalts. Geochemistry, Geophysics, Geosystems 16, 719-735.

Parai, R., Mukhopadhyay, S. (2018) Xenon isotopic constraints on the history of volatile recycling into the mantle. Nature 560, 223-227.

PARAi, R., MukHOPADHYAy, S., Standish, J.J. (2012) Heterogeneous upper mantle $\mathrm{Ne}$, Ar and Xe isotopic compositions and a possible Dupal noble gas signature recorded in basalts from the Southwest Indian Ridge. Earth and Planetary Science Letters 359-360, 227-239.

PepIN, R.O. (2003) On Noble Gas Processing in the Solar Accretion Disk. Space Science Reviews 106, 211-230.

Petö, M.K., Mukhopadhyay, S., Kelley, K.A. (2013) Heterogeneities from the first 100 million years recorded in deep mantle noble gases from the Northern Lau Back-arc Basin. Earth and Planetary Science Letters 369-370, 13-23.

PoredA, R.J., FARLEY, K.A. (1992) Rare gases in Samoan xenoliths. Earth and Planetary Science Letters 113, 129-144.

Pujol, M., Marty, B., Burgess, R. (2011) Chondritic-like xenon trapped in Archean rocks: A possible signature of the ancient atmosphere. Earth and Planetary Science Letters 308, 298-306.

Smye, A.J., JaCKson, C.R.M., Konrad-Schmolke, M., Hesse, M.A., Parman, S.W., Shuster, D.L., Ballentine, C.J. (2017) Noble gases recycled into the mantle through cold subduction zones. Earth and Planetary Science Letters 471, 65-73.

Trieloff, M., Kunz, J., Clague, D.A., Harrison, D., Allègre, C.J. (2000) The Nature of pristine noble gases in mantle plumes. Science $288,1036-1038$

Trieloff, M., KunZ, J., Allègre, C.J. (2002) Noble gas systematics of the Reunion mantle plume source and the origin of primordial noble gases in Earth's mantle. Earth and Planetary Science Letters 200, 297-313.

Tucker, J.M., Mukhopadhyay, S., Schilling, J.-G. (2012) The heavy noble gas composition of the depleted MORB mantle (DMM) and its implications for the preservation of heterogeneities in the mantle. Earth and Planetary Science Letters 355-356, 244-254.

VAN KeKen, P.E., Hacker, B.R., SYracuse, E.M., Abers, G.A. (2011) Subduction factory: 4. Depth dependent flux of $\mathrm{H}_{2} \mathrm{O}$ from subducting slabs worldwide. Journal of Geophysical Research: Solid Earth 116. 